\section{News from DMCCB - Targeted Protein Degradation}

\section{Georg Jaeschke}

*Correspondence: Dr. G. Jaeschke, F. Hoffmann-La Roche Ltd., Medicinal Chemistry, Pharma Research and Early Development, Innovation Center Basel, Grenzacherstrasse 124, 4070 Basel, Switzerland; E-mail: georg.jaeschke@roche.com

Keywords: Anticancer drug discovery · E3 ligases · PROTACs · Proteasome $\cdot$ Targeted protein degradation

Targeted protein degradation is a new therapeutic concept within medicinal chemistry that has the promise to expand the scope of what can be achieved with small molecules. The approach relies on the use of bifunctional molecules that can bind simultaneously to the protein of interest (POI) and an E3 ligase such as CRBN (Cereblon) or VHL (Von Hippel Lindau). In the ternary complex which is formed, the POI is positioned in proximity to the E3 ligase, which can lead to ubiquitination of the POI. The polyubiquitinylated protein is thereby flagged for decomposition and can be recognized and degraded by the proteasome upon dissociation from the ternary complex (see Fig. 1). The heterobifunctional molecules used are often also referred to as PROTACs (proteolysis targeting chimeras). Possible advantages compared to traditional approaches in medicinal chemistry have been outlined in multiple reviews ${ }^{[1]}$ and include:

- Potential to address so far undruggable targets (e.g. transcription factors) or functions of a POI (e.g. scaffolding effects of kinases) ${ }^{[2]}$

- Possibility to have higher selectivity compared to the starting ligand used for binding to the $\mathrm{POI}^{[3]}$
- Catalytic mode of action as a PROTAC can potentially degrade more than a single protein ${ }^{[4]}$

- Transient interaction with the target might be sufficient to achieve efficacy

On the other hand, degrader molecules are characterized by a high molecular weight which typically ranges from 700 to 1000 $\mathrm{Da}$, translating into challenges to achieve sufficient permeability and oral bioavailability. For oncology indications, there are also risks associated with the development of resistance mechanisms which may be dependent on the E3 ligase as well as the target protein.

The first experimental example of targeted protein degradation was published in 2001 but the utility of initial compounds was hampered by large peptidic E3 binders leading to unfavorable physicochemical properties. ${ }^{[5]}$ The first in vivo active molecules $^{[6]}$ were published only in 2015 and a key driver for this recent progress was the use of potent and small E3 ligase binders that target either CRBN or VHL E3 ligases conferring improved degrader properties. As a consequence, the interest in the area has grown significantly and the concept has been rapidly expanded to multiple targets including RIPK $2,{ }^{[7]} \mathrm{BCR}-\mathrm{ABL},{ }^{[8]} \mathrm{FKBP} 12,{ }^{[6 a]}$ $\mathrm{ERRa}^{[9]} \mathrm{AR}^{[10]}$ and bromodomains ${ }^{[3 \mathrm{~b}, 6 \mathrm{~b}, 11]}$ (for some representative examples see Fig. 2).

So what is next? Advancing degrader molecules to the clinic for several targets is needed to ultimately prove the concept and development activities for some targets including AR degraders are quite advanced. However, optimizing degrader molecules that have successfully demonstrated an in vitro proof of concept to clinical candidates is a complex and challenging task and recent publications showcase concepts that might be helpful in these efforts:

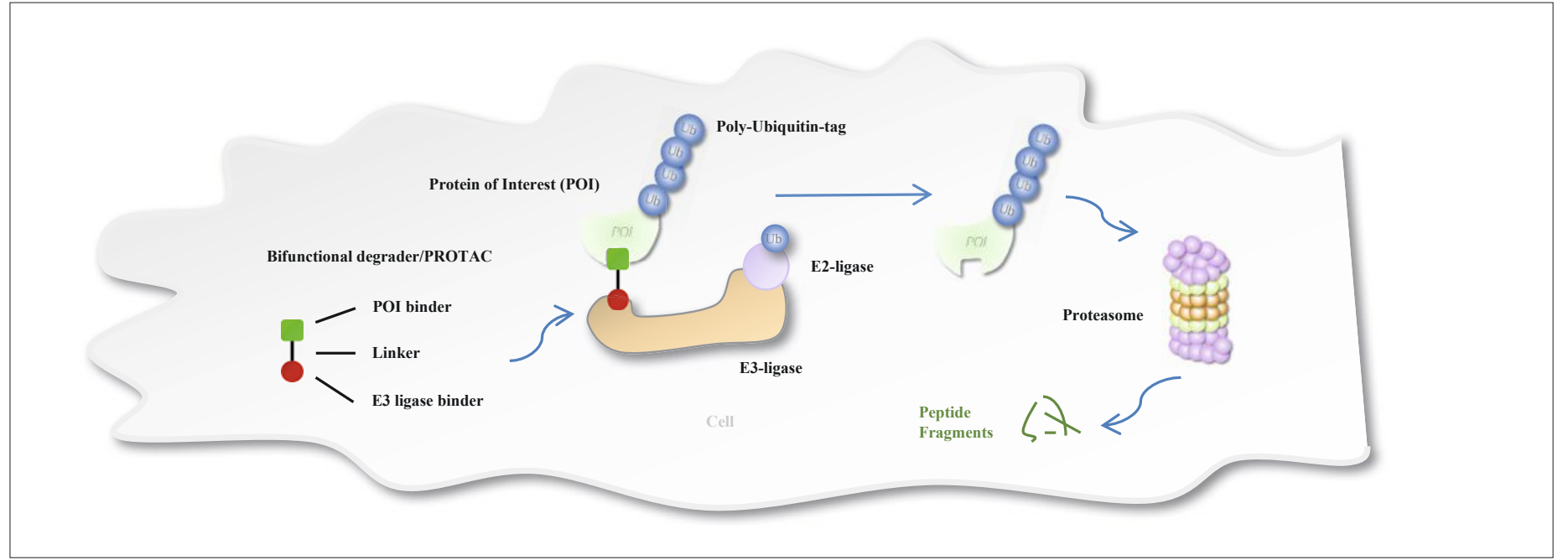

Fig. 1. Bifunctional degrader molecule forms a ternary complex with $\mathrm{POI}$, which is not a natural substrate of the E3-ligase, and the E3 ligase. Ubiquitin moieties are transferred to the $\mathrm{POI}$ and the polyubiquitinylated $\mathrm{POI}$ is recognized by the proteasome and degraded. The degrader molecule is available for another catalytic cycle. 


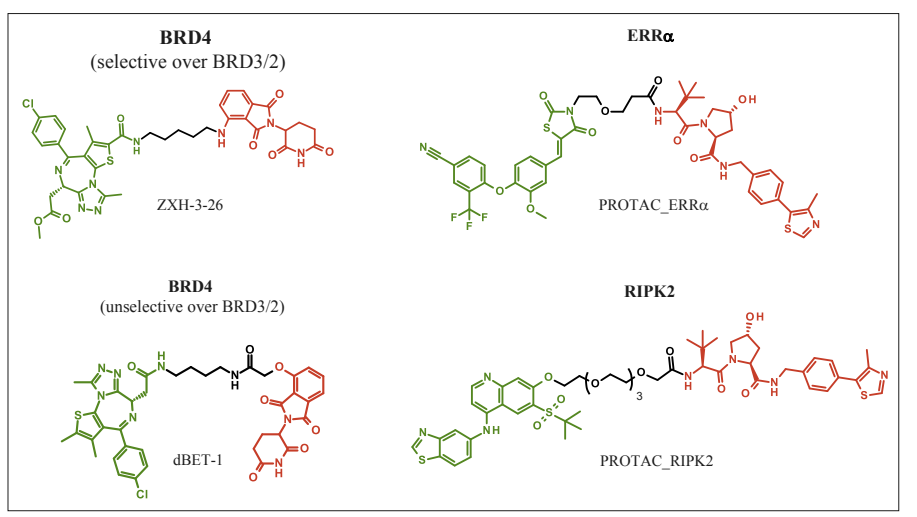

Fig. 2. Examples of bifunctional molecules that in vitro degrade different target proteins. The E3-binding part depicted in red recruits either cereblon or VHL E3 ligases.

\section{Optimizing Ternary Complex Formation}

$\mathrm{X}$-ray structures of ternary complexes using BRD4 degrader molecules with CRBN as E3 ligase have been reported recently. ${ }^{[3 \mathrm{~b}]}$ Interestingly, the authors do not observe a positive cooperativity between the two proteins suggesting that attractive proteinprotein interactions are not mandatory for efficient degradation and this finding points towards a broad applicability of this concept to various targets. Analysis of the ternary complex led to the identification of degraders with favorable and more 'drug-like' properties and revealed underlying interprotein contacts distant from the immediate binding site that could be utilized to design BRD4 selective degraders such as ZXH-3-26 (Fig. 2) able to discriminate between the closely homologous BRD3 and BRD2. A better understanding of ternary complexes is therefore expected to provide valuable information to guide medicinal chemists in optimizing linker length and trajectory in a rational manner in order to improve potency and selectivity. ${ }^{[12]}$

\section{Understanding the Kinetics of Targeted Protein Degradation}

Effective formation of a ternary complex is considered to be essential for targets where the ubiquitination of the POI is slow compared to the reversible formation of the ternary complex. It is also conceivable that the ubiquitination is not the rate-limiting step leading to systems which follow a 'hit and run' model where very low levels of a ternary complex are sufficient to induce robust protein degradation. ${ }^{[13]}$ To guide medicinal chemistry efforts new tools will be required to better characterize the complex biological events and kinetic processes leading to degradation.

\section{Utilizing Improved E3 Binders of CRBN, VHL or other E3 Ligases}

The rapid progress of the identification of small, potent E3 binders indicates that improvement in this area has the potential to further advance this technology. As more than 600 E3 ligases are known, the identification of new binders for the therapeutically untapped reservoir of E3 ligases is a vector for future innovation in the field of targeted protein degradation. In addition, intense focus by the medicinal chemistry community might lead to improved CRBN and VHL binders with enhanced degradation potency, catalytic efficiency as well as physicochemical properties.

In summary, progress has been made on multiple fronts, and while vast optimization potential exists, the field is expected to accelerate towards identification of candidates that can be advanced into clinical studies. Progress in the area of targeted protein degradation is certainly closely watched by the small molecule drug discovery community and expectations remain high that this fascinating new technology will further equip the armory of medicinal chemistry and provide new hope for patients in need.

Received: October 25, 2018

[1] a) I. Churcher, J. Med. Chem. 2018, 61, 444, DOI: 10.1021/acs. jmedchem.7b01272; b) J. Salami, C. M. Crews, Science 2017, 355, 1163, DOI: $10.1126 /$ science.aam 7340 .

[2] G. M. Burslem, B. E. Smith, A. C. Lai, S. Jaime-Figueroa, D. C. McQuaid, D. P. Bondeson, M. Toure, H. Dong, Y. Qian, J. Wang, A. P. Crew, J. Hines, C. M. Crews, Cell Chem. Biol. 2018, 25, 67, DOI: 10.1016/j. chembiol.2017.09.009.

[3] a) D. P. Bondeson, B. E. Smith, G. M. Burslem, A. D. Buhimschi, J. Hines, S. Jaime-Figueroa, J. Wang, B. D. Hamman, A. Ishchenko, C. M. Crews, Cell Chem. Biol. 2018, 25, 78, DOI: 10.1016/j.chembiol.2017.09.010; b) R. P. Nowak, S. L. DeAngelo, D. Buckley, Z. He, K. A. Donovan, J. An, N. Safaee, M. P. Jedrychowski, C. M. Ponthier, M. Ishoey, T. Zhang, J. D. Mancias, N. S. Gray, J. E. Bradner, E. S. Fischer, Nat. Chem. Biol. 2018, 1552, DOI: 10.1038/s41589-018-0055-y; c) M. S. Gadd, A. Testa, X. Lucas, K. H. Chan, W. Chen, D. J. Lamont, M. Zengerle, A. Ciulli, Nat. Chem. Biol. 2017, 13, 514, DOI: 10.1038/nchembio.2329.

[4] D. P. Bondeson, A. Mares, I. E. D. Smith, E. Ko, S. Campos, A. H. Miah, K. E. Mulholland, N. Routly, D. L. Buckley, J. L. Gustafson, N. Zinn, P. Grandi, S. Shimamura, G. Bergamini, M. Faelth-Savitski, M. Bantscheff, C. Cox, D. A. Gordon, R. R. Willard, J. J. Flanagan, L. N. Casillas, B. J. Votta, W. den Besten, K. Famm, L. Kruidenier, P. S. Carter, J. D. Harling, I. Churcher, C. M. Crews, Nat. Chem. Biol. 2015, 11, 611, DOI: 10.1038/ nchembio. 1858 .

[5] K. M. Sakamoto, K. B. Kim, A. Kumagai, F. Mercurio, C. M. Crews, R. J. Deshaies, Proc. Natl. Acad. Sci. USA 2001, 98, 8554, DOI: 10.1073/ pnas. 141230798 .

[6] a) G. E. Winter, D. L. Buckley, J. Paulk, J. M. Roberts, A. Souza, S. DhePaganon, J. E. Bradner, Science 2015, 348, 1376, DOI: 10.1126/science. aab1433; b) J. Lu, Y. Qian, M. Altieri, H. Dong, J. Wang, K. Raina, J. Hines, J. D. Winkler, A. P. Crew, K. Coleman, C. M. Crews, Chem. Biol. 2015, 22, 755, DOI: 10.1016/j.chembiol.2015.05.009.

[7] D. P. Bondeson, C. M. Crews, 'Targeted Protein Degradation by Small Molecules', Vol. 57, Annual Reviews Inc., 4139 El Camino Way, P.O. Box 10139, Palo Alto, CA 94306, United States, 2017, pp. 107-23, DOI: 0.1146/ annurev-pharmtox-010715-103507.

[8] A. C. Lai, M. Toure, D. Hellerschmied, J. Salami, S. Jaime-Figueroa, E. Ko, J. Hines, C. M. Crews, Angew. Chem., Int. Ed. 2016, 55, 807, DOI: 10.1002/ anie. 201507634.

[9] J. J. Flanagan, Y. M. Qian, S. M. Gough, M. Andreoli, M. Bookbinder, J. Bradley, E. Rousseau, R. Willard, C. M. Crews, A. P. Crew, I. Taylor, J. Houston, Cancer Res. 2018, 78, 4, DOI: 10.1158/15387445. sabcs17-p4-04-04.

[10] J. Salami, S. Alabi, R. R. Willard, N. J. Vitale, J. Wang, H. Dong, M. Jin, D. P. McDonnell, A. P. Crew, T. K. Neklesa, C. M. Crews, Commun. Biol. 2018, 1, 100, DOI: 10.1038/s42003-018-0105-8.

[11] M. Zengerle, K.-H. Chan, A. Ciulli, ACS Chem. Biol. 2015, 10, 1770, DOI: 10.1021/acschembio.5b00216.

[12] S. J. Hughes, A. Ciulli, Essays Biochem. 2017, 61, 505, DOI: 10.1042/ EBC20170041.

[13] S. L. Fisher, A. J. Phillips, Curr. Opin. Chem. Biol. 2018, 44, 47, DOI: 10.1016/j.cbpa.2018.05.004. 